\title{
THE ESA-EVE POLARIZATION LIDAR FOR ASSESSING THE AEOLUS AEROSOL PRODUCT PERFOMANCE
}

\author{
Peristera Paschou ${ }^{* 1,2}$, Emmanouil Proestakis ${ }^{1}$, Alexandra Tsekeri ${ }^{1}$, Nikos Siomos ${ }^{2}$, Anto- \\ nis Gkikas ${ }^{1}$, Anna Gialitaki ${ }^{1}$, Eleni Marinou ${ }^{1,3}$, Ioannis Binietoglou ${ }^{4}$, Charikleia Meleti ${ }^{2}$, \\ Volker Freudenthaler ${ }^{5}$, George Georgoussis ${ }^{6}$, George Doxastakis ${ }^{6}$, Alexandros Louri- \\ das $^{6}$, Jonas Von Bismarck ${ }^{7}$ and Vassilis Amiridis ${ }^{1}$ \\ ${ }^{1}$ IAASARS, National Observatory of Athens, Athens, 15236, Greece \\ ${ }^{2}$ Aristotle University of Thessaloniki, Greece \\ ${ }^{3}$ Institut für Physik der Atmosphäre, Deutsches Zentrum für Luft und Raumfahrt, Germany \\ ${ }^{4}$ National Institute of R\&D for Optoelectronics, Romania \\ ${ }^{5}$ Ludwig-Maximilians-Universitat, Germany \\ ${ }^{6}$ Raymetrics S.A., Greece \\ ${ }^{7}$ Directorate of Earth Observation Programmes, ESA-ESRIN, Italy
}

"Email:pepaschou@noa.gr

\begin{abstract}
We present the EVE lidar concept, a combined linear/circular polarization system, tailored to evaluate the spaceborne ALADIN Doppler lidar system aerosol retrievals. EVE, currently under development, aims to provide the ESA-Aeolus mission with a flexible, mobile reference ground-based lidar system capable of providing well-characterized fiducial reference measurements of aerosol optical properties. Since ALADIN detects only the copolar component of the backscattered circularly polarized radiation, a portion of the received radiation gets lost, leading to an underestimation of the backscatter coefficient and the circular depolarization ratio in strongly depolarizing scenes with nonspherical particles. The main focus of the new EVE lidar is to quantify these uncertainties and to evaluate aerosol backscatter/extinction retrievals for Aeolus, and later also for EarthCARE product validation, quality assessment and improvement.
\end{abstract}

\section{INTRODUCTION}

The validation and calibration procedures of advanced spaceborne earth observation instruments have traditionally relied on well-calibrated ground-based instruments to provide fiducial reference measure- ments, capable for assessing the performance of the space sensors [1]-[3]. The atmospheric missions of the European Space Agency (ESA), and among them the Earth Explorers Aeolus [4] and EarthCARE [5], are no exceptions, particularly with respect to the validation of profile products related to aerosols and clouds.

The Atmospheric LAser Doppler INstrument (ALADIN) on board Aeolus, is designed to provide global profiles of the Horizontal Line-Of-Sight (HLOS) wind component in the troposphere and the lower stratosphere [4], [6]. ALADIN is a sophisticated Doppler Wind Lidar (DWL) operating in the ultraviolet region of the spectrum (355 $\mathrm{nm})$, implemented in a transceiver configuration and tilted $35^{\circ}$ from nadir [7]. It is also a High Spectral Resolution Lidar (HSRL), capable of deriving aerosol optical parameters such as particle backscatter and extinction coefficient profiles. Furthermore, the EarthCARE future mission of ESA is designed to utilize an array of scientific instruments, including the ATmospheric LIDar, ATLID. ATLID is an HSRL, operating at the wavelength of $355 \mathrm{~nm}$ with a high-spectral resolution receiver and circular depolarization channel, that will provide vertically resolved optical products for aerosol and thin clouds.

Both ALADIN and ATLID have an identified need for co-located measurements with 
ground-based lidars operating at the same wavelength $(355 \mathrm{~nm})$ for the data product's assessment and validation. While a growing fraction of the ground-based lidar systems operate a depolarization channel at $355 \mathrm{~nm}$, the common lidar types featured for example in the European Aerosol Research Lidar Network (EARLINET), do not perform circular depolarization measurements alongside linear depolarization measurements [8].

The main aim of EVE (Enhancement and Validation of ESA products) lidar is to provide the upcoming ESA missions of Aeolus and EarthCARE, with a well-characterized, mobile ground-based lidar system, capable of providing fiducial reference measurements for ALADIN and ATLID.

\section{AEOLUS AEROSOL PRODUCT}

The aerosol and cloud spin-off product of Aeolus comprises of profiles of extinction coefficient, backscatter coefficient, and extinction-to-backscatter (lidar) ratio at 355 nm. An inherent weakness of ALADIN is the lack of detection of the cross-polarized return of the emitted circularly-polarized signal. This limitation has almost negligible impact when near-spherical aerosol particles are probed. However, when non-spherical, depolarizing particles, such as desert dust, volcanic ash, and ice crystals are encountered along the Line-of-Sight (LoS) of ALADIN, the retrievals will deviate from those obtained by an instrument that would measure both the co-polar and the cross-polar components. The deviation of the measured backscatter coefficient by ALADIN (middle panel), with respect to the corresponding amount, which would have been measured if cross- and co-polar components were taken into account (left panel), is depicted in Figure 1 for a case of nonspherical dust particles.

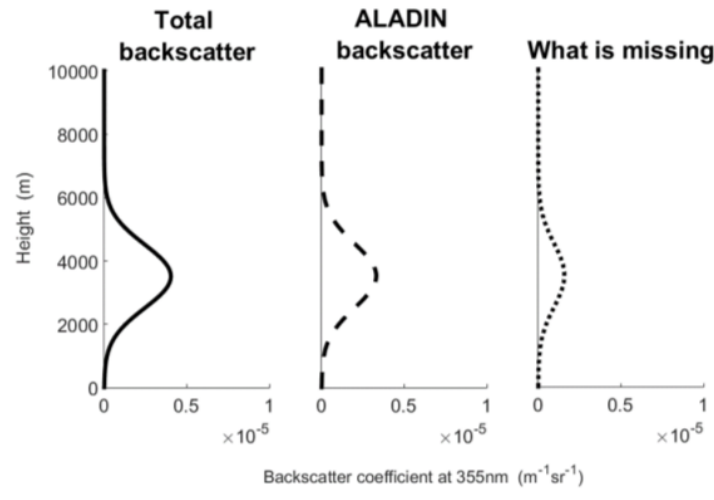

Figure 1: Total backscatter coefficient of dust at 355 $\mathrm{nm}$ as a function of altitude (left), the corresponding co-polar component detected by ALADIN (middle) and the undetected-missing cross-polar component (right)

The underestimation of the total backscatter coefficient retrieved by ALADIN, due to the detection only of the co-polar component, compared to the total backscatter when both cross- $(\mathrm{b} \perp)$ and co-polar $(\mathrm{b} \|)$ components would be measured, is depicted in Figure 2. For this estimation, we used CALIPSO (Cloud-Aerosol Lidar and Infrared Pathfinder Satellite Observation; [9]), based on the LIVAS (LIdar climatology of Vertical Aerosol Structure for space-based lidar simulation studies) threedimensional aerosol and cloud database [10]. More specifically, based on CALIPSO Level 2 version 3 aerosol products we calculated the profiles of linear depolarization ratio at $532 \mathrm{~nm}$, and accordingly, converted them to $355 \mathrm{~nm}$. Considering the direct relationship between linear and circular depolarization for randomly oriented particles $\left(\delta_{\mathrm{C}}=2 \delta_{\mathrm{L}} /\left(1-\delta_{\mathrm{L}}\right)\right)$ [11], we calculated the circular depolarization profiles at $355 \mathrm{~nm}\left(\delta_{\mathrm{C}}=\mathrm{b} \perp / \mathrm{b} \|\right)$ and computed the average underestimation of the total aerosol backscatter due to the detection of only the co-polar component ($100 \cdot b \perp /(b \|+b \perp))$. On a global scale, the underestimation of the average backscatter coefficient could be up to $65 \%$. Similarly, Wandinger et al. [12] in their presentation in the "ADM-Aeolus Science and $\mathrm{Cal} / \mathrm{Val}$ Workshop" showed that the underestimation of the backscatter coefficient can be up to $50 \%$ for desert dust and up to $70 \%$ for cirrus. 


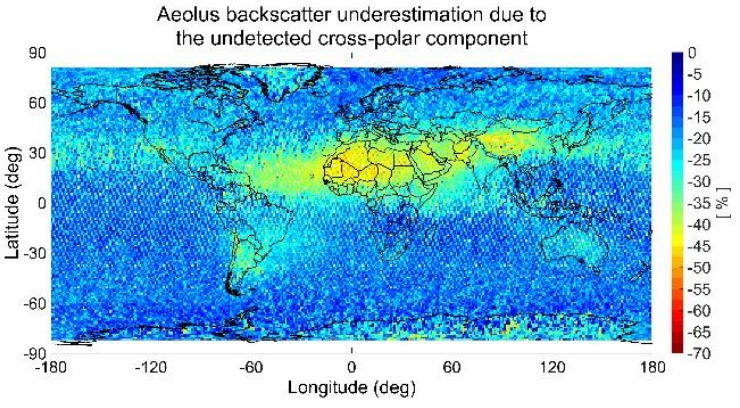

Figure 2: Global-scale underestimation of the averaged total backscatter coefficient at $355 \mathrm{~nm}$ for the year 2007, due to detection of only the co-polar component, based on LIVAS database results.

Furthermore, polarization-dependent multiple scattering effects, especially in the case of cirrus clouds, can significantly bias the retrievals for both extinction and backscatter coefficients. Since the EVE lidar will measure both linearly and circularly polarized signals, the validation of the relation between circular and linear depolarization ratios for randomly oriented particles could be used as indicator of the multiple scattering effects [13].

Orientation of the particles may also bias the retrievals [14] mainly for the backscatter coefficient and less for the extinction coefficient as shown in the simulations in Figure 3. The Figure 3 shows that the extinction and backscatter coefficients of randomly oriented particles (black line) are different than the extinction and backscatter coefficients of oriented particles (blue and red lines). Moreover, the latter depend on the viewing angle, thus, in case of oriented particles a zenith-looking ground-based lidar will measure different values (blue line) than ALADIN, looking at $35^{\circ}$ (red line). EVE is optimally designed to reproduce the ALADIN measurements at $35^{\circ}$. It will also have the capability to perform measurements at multiple angles and provide indicators of particle orientation.
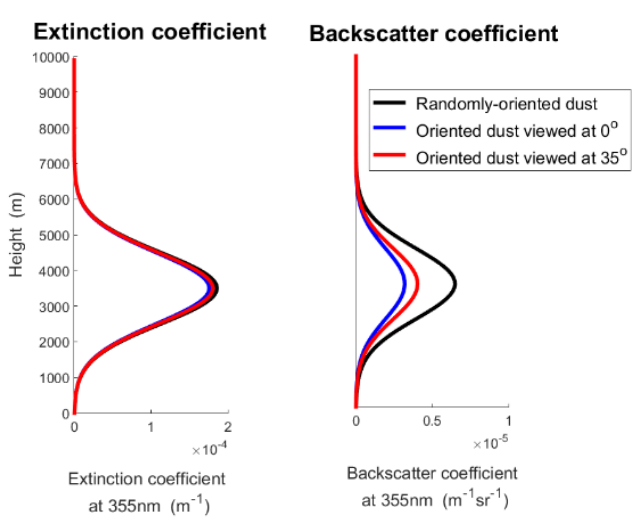

Figure 3: Simulations of the extinction coefficient (left) and backscatter coefficient at $355 \mathrm{~nm}$ (right) as a function of altitude for a layer of randomly oriented (black line) and vertically oriented particles (blue and red lines). For oriented particles, the extinction and backscatter coefficients viewed with a lidar at $0^{\circ}$ are denoted with blue and viewed at $35^{\circ}$ with red.

\section{EVE CONCEPTUAL DESIGN}

The system design will utilize a dual-telescope/dual-laser configuration, operating in the UV at $355 \mathrm{~nm}$, and will allow the adjustment of the zenith angle from 3 to 35 degrees and full adjustment of the azimuth angle to fulfil the requirements of both Aeolus and EarthCARE. The overall design will also allow for an easy integration and operation of additional emission/receiver channels (for example at $532 \mathrm{~nm}$ and 1064 $\mathrm{nm}$ for aerosol characterization) at a later stage, and it enables the transportation to and participation in Aeolus (and EarthCARE) CalVal campaign activities, such as the upcoming ASKOS tropical campaign for the evaluation of Aeolus aerosol products. The conceptual design for the EVE mobile lidar is presented in Figure 4.

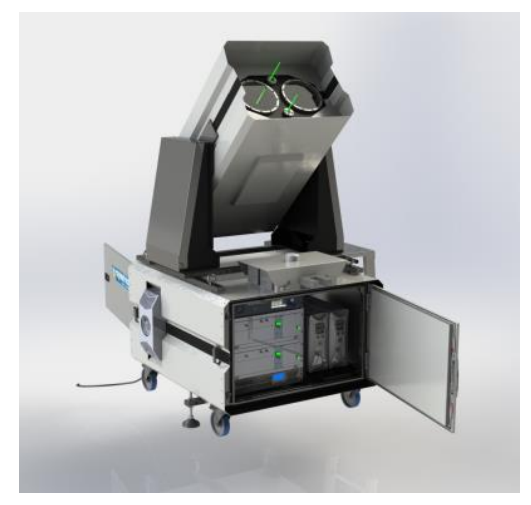

Figure 4: Conceptual design for the EVE mobile lidar. The system measurements are based on two lasers emitting circularly and linearly polarized radiation at $355 \mathrm{~nm}$ and two receiving telescopes. It will be 
easily transportable to facilitate CalVal operations at a variety of sites and aerosol/cloud conditions.

First, we aim to develop a lidar simulator to demonstrate the optimal measurement strategy of EVE for the Aeolus CalVal applications. In the test phase, EVE will be operated under different atmospheric environments and conditions in order to refine the prototype design, to demonstrate its fitness for purpose and conformity with the design specifications. As such, the testing and validation of the early prototype version of the EVE lidar includes the operation alongside a quality assured local lidar system for a period of at least one month, for intercomparison and performance evaluation purposes. This intercomparison will be performed at the PANhellenic GEophysical observatory of Antikythera (PANGEA), a fixed observational platform of NOA (National Observatory of Athens) at the island of Antikythera-Greece. PANGEA hosts the multi-wavelength lidar system PollyXT-NOA (portable aerosol Raman lidar system with eXTended capabilities; [15]) and the AERONET's photometer. The observatory location is considered representative of the broader region in the Mediterranean as it is a crossroad of different aerosol pathways, significantly impacted by mineral dust from Africa, smoke from frequent forest fires and agricultural biomass burning, marine particles, as well as anthropogenic background pollution from megacities such as Athens and Istanbul [16].

In parallel, we will develop the processing algorithms for the retrieval of the lidar optical products. The processing algorithms will provide the attenuated backscatter, circular and linear depolarization ratios, backscatter coefficient and extinction coefficient at $355 \mathrm{~nm}$, including the associated uncertainties.

\section{ACKNOWLEDGEMENTS}

EVE is implemented by NOA and Raymetrics S.A. under an ESA contract. We acknowledge the support of (a) the project "PANhellenic infrastructure for Atmospheric Composition and climatE change" (MIS 5021516) which is implemented under the Action "Reinforcement of the Research and Innovation Infrastructure", funded by the Operational Programme "Competitiveness, Entrepreneurship and Innovation" (NSRF 2014-2020) and co-financed by Greece and the European Union (European Regional Development Fund); (b) the European Research Council under the European Community's Horizon 2020 research and innovation framework program/ERC Grant Agreement 725698 (DTECT) and (c) the Stavros Niarchos Foundation.

\section{REFERENCES}

[1] L. Mona et al., Atmos. Chem. Phys., 2009.

[2] G. Pappalardo et al., J. Geophys. Res. Atmos., 2010.

[3] E. Proestakis et al., Atmos. Chem. Phys. Discuss., pp. 1-30, Feb. 2019.

[4] A. Stoffelen et al., Q. J. R. Meteorol. Soc., vol. 132, no. 619, pp. 1927-1947, Jul. 2006.

[5] A. J. Illingworth et al., Bull. Am. Meteorol. Soc., 2015.

[6] A. Dabas, Comptes Rendus Geosci., vol. 342, no. 4-5, pp. 370-379, Apr. 2010.

[7] S. Lolli et al., Atmos. Meas. Tech., vol. 6, no. 12, pp. 3349-3358, Dec. 2013.

[8] G. Pappalardo et al., Atmos. Meas. Tech., vol. 7, no. 8, pp. 2389-2409, Aug. 2014.

[9] D. M. Winker et al., Bull. Am. Meteorol. Soc., 2010.

[10] V. Amiridis et al., Atmos. Chem. Phys., vol. 15, no. 13, pp. 7127-7153, Jul. 2015.

[11] M. I. Mishchenko and J. W. Hovenier, Opt. Lett., vol. 20, no. 12, p. 1356, Jun. 1995.

[12] U. Wandinger et al., in ADM-Aeolus Science and CAL/VAL Workshop, ESA-ESRIN, 2015.

[13] G. Roy and N. Roy, Appl. Opt., 2008.

[14] B. V. Kaul et al., Appl. Opt., vol. 43, no. 36, p. 6620, Dec. 2004.

[15] R. Engelmann et al., Atmos. Meas. Tech., 2016.

[16] E. Marinou et al., Atmos. Chem. Phys., 2017. 